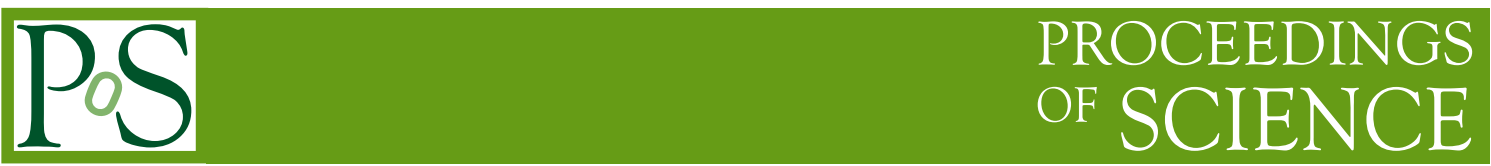

\title{
Perturbative lattice artefacts in the SF coupling for technicolor-inspired models
}

\author{
Stefan Sint* and Pol Vilaseca \\ School of Mathematics, Trinity College, Dublin 2, Ireland \\ E-mail: sint@maths.tcd.ie, pol@maths.tcd.ie
}

\begin{abstract}
Viable candidate theories for electroweak dynamical symmetry breaking are expected to show (near) conformal behaviour in order to accommodate current phenomenological constraints. In principle, renormalisation group studies using finite volume renormalisation schemes are wellsuited to verify this property in a given model. The most practical schemes are based on the Schrödinger functional (SF), but suffer from potentially large O(a) effects. Some care has to be taken to remove these effects and to set up a scheme where cutoff effects are small and under control. We here take a step in this direction by analysing various set-ups for the SF coupling at one-loop order in perturbation theory.
\end{abstract}

The XXIX International Symposium on Lattice Field Theory - Lattice 2011

July 10-16, 2011

Squaw Valley, Lake Tahoe, California

\footnotetext{
*Speaker.
} 


\section{Introduction}

The prospect of discovering the underlying dynamics of electroweak symmetry breaking at the LHC has triggered new efforts to check the viability of technicolor-inspired models of dynamical symmetry breaking (see e.g. [1]). It is widely believed that a viable model should show conformal or near conformal behaviour over some range of scales. Such behaviour is expected for some QCD inspired models with certain values of $\left(N_{\mathrm{f}}, N\right)$, where $N_{\mathrm{f}}$ denotes the number of fermion flavours and $N$ the number of colours. Furthermore, one needs to choose the representation of the gauge group for the fermions. We will here focus on the example of $N_{\mathrm{f}}=2$ flavours in the two-index symmetric (sextet) representation of the colour group SU(3).

Non-perturbative studies of the renormalization group evolution of the coupling provide a direct way to test for (near) conformal behaviour. Finite volume renormalization schemes based on the Schrödinger functional (SF) [2, 3] have become popular in this context (cf. [4] for a recent review). However, some care has to be taken to distinguish universal continuum properties from lattice artefacts. Before embarking on a non-perturbative study it is thus advisable to test any proposed framework in perturbation theory, where the continuum limit is known and the size of lattice artefacts can be assessed.

In this contribution we propose a closer look at the set-up of the SF with Wilson fermions. As is well-known from lattice QCD, it is important to remove lattice effects linear in $a$, which originate both from the time boundaries and from the bulk [5]. The bulk $\mathrm{O}(a)$ effects can be completely eliminated by non-perturbative Symanzik improvement [6], and for fermions in higher $\mathrm{SU}(N)$ representations this has been attempted in ref. [7]. An alternative consists in using the chirally rotated $\mathrm{SF}(\chi \mathrm{SF})[8,9]$, which implements the mechanism of automatic $\mathrm{O}(a)$ improvement. We here compare both options, and our study also provides a further test of the $\chi \mathrm{SF}$ in perturbation theory.

Even if the leading $\mathrm{O}(a)$ effects are eliminated, the size of the remaining higher lattice artefacts in the coupling can be large, and in some cases the asymptotic $\mathrm{O}\left(a^{2}\right)$ behaviour sets in rather late, beyond the typical lattice sizes accessible to simulations [10]. While we only provide details for fundamental and the sextet representations of SU(3), our observations are generic and thus also relevant for gauge group SU(2) and fermions in other other non-fundamental representations [14, $11]$.

This writeup is organized as follows: after a short reminder of the basic definitions of the SF coupling we discuss the parameterization of cutoff effects in the step-scaling function and criteria for assessing their size. We then discuss the perturbative one-loop data before we draw our conclusion and outline future directions.

\section{The SF coupling}

The Schrödinger functional provides us with a mass-independent finite volume scheme for the coupling. It is defined through the variation of a colour-electric background field $B_{\mu}$ with respect to a parameter $\eta$. Denoting its effective action by $\Gamma[B]$, the coupling is defined by $[12,13]$,

$$
\bar{g}^{2}(L)=\left.\frac{\partial_{\eta} \Gamma_{0}[B]}{\partial_{\eta} \Gamma[B]}\right|_{\eta=0}, \quad \Gamma[B] \stackrel{g_{0} \rightarrow 0}{\sim} \frac{1}{g_{0}^{2}} \Gamma_{0}[B]+\Gamma_{1}[B]+\ldots
$$


At one-loop order in perturbation theory its relation to the bare coupling $g_{0}$ is of the form,

$$
\bar{g}^{2}(L)=g_{0}^{2}+p_{1}(L / a) g_{0}^{4}+\ldots, \quad p_{1}(L / a)=p_{1,0}(L / a)+N_{\mathrm{f}} p_{1,1}(L / a) .
$$

Although the SF coupling is an observable in numerical simulations, it is expensive to measure and simulations with Wilson fermions are typically limited to lattices with $L / a \leq 16$. It is therefore important to find SF schemes where cutoff effects are as small as possible and where the asymptotic regime is reached already on such relatively small lattices.

\section{Parameterization of cutoff effects}

In the traditional analysis of lattice QCD data the quantity of interest is the step-scaling function (SSF),

$$
\Sigma(u, a / L)=\left.\bar{g}^{2}(2 L)\right|_{u=\bar{g}^{2}(L)}=u+\Sigma_{1}(a / L) u^{2}+\mathrm{O}\left(u^{3}\right) .
$$

It has a universal continuum limit,

$$
\lim _{a \rightarrow 0} \Sigma(u, a / L)=\sigma(u)=u+\sigma_{1} u^{2}+\mathrm{O}\left(u^{3}\right), \quad \sigma_{1}=2 b_{0} \ln (2),
$$

where the leading term contains the one-loop coefficient of the $\beta$-function,

$$
b_{0}=b_{0,0}+N_{\mathrm{f}} b_{0,1}=\frac{1}{16 \pi^{2}}\left(\frac{11 N}{3}-N_{\mathrm{f}} \frac{4}{3} T_{R}\right),
$$

with $T_{R}=1 / 2, N,(N+2) / 2$ for $R$ denoting the fundamental, adjoint and two-index symmetric representations, respectively. We now define relative deviations from pure gauge and fermionic continuum coefficients in $\sigma_{1}=\sigma_{1,0}+N_{\mathrm{f}} \sigma_{1,1}$ :

$$
\delta_{1,0}(a / L)=\frac{\Sigma_{1,0}(a / L)-\sigma_{1,0}}{\sigma_{1,0}}, \quad \delta_{1,1}(a / L)=\frac{\Sigma_{1,1}(a / L)-\sigma_{1,1}}{\sigma_{1,1}} .
$$

How large can we allow the cutoff effects to be? It is instructive to study this question at fixed lattice size: a relative deviation of $\delta_{1,1}=100 \%$ could then be mistaken for getting $N_{\mathrm{f}}$ wrong by a factor 2 , since

$$
N_{\mathrm{f}} \Sigma_{1,1}=\left(1+\delta_{1,1}\right) N_{\mathrm{f}} \sigma_{1,1} \approx 2 N_{\mathrm{f}} \sigma_{1,1} .
$$

Hence, with all parameters fixed, requiring $\left|\delta_{1,1}(a / L)\right| \ll 1$ seems reasonable and sets a lower limit on $L / a$. For comparison, the relative deviation in the pure $\mathrm{SU}(3)$ gauge theory on the smallest lattices is given by $\delta_{1,0}(1 / 4)=-20 \%, \delta_{1,0}(1 / 6)=-8 \%$ and $\delta_{1,0}(1 / 8)=-4 \%$, and thus quickly approaches zero. It seems natural to require a similar behaviour for the contribution from the fermions.

\section{4. $\mathbf{O}(a)$ vs. $\mathbf{O}\left(a^{2}\right)$ asymptotic behaviour}

$\mathrm{O}(a)$ effects in the SSF with the standard SF originate from both the bulk and the boundaries. Boundary $\mathrm{O}(a)$ effects can be cancelled by the local boundary counterterms $\propto c_{\mathrm{t}}, \tilde{c}_{\mathrm{t}}$ [5]. The only bulk counterterm is the clover term with coefficient $c_{\mathrm{sw}}$. With the $\chi \mathrm{SF}$ only boundary $\mathrm{O}(a)$ counterterms are required $\left(\propto c_{\mathrm{t}}, d_{s}\right)$ [8]. The price to be paid for this advantage consists in the tuning 

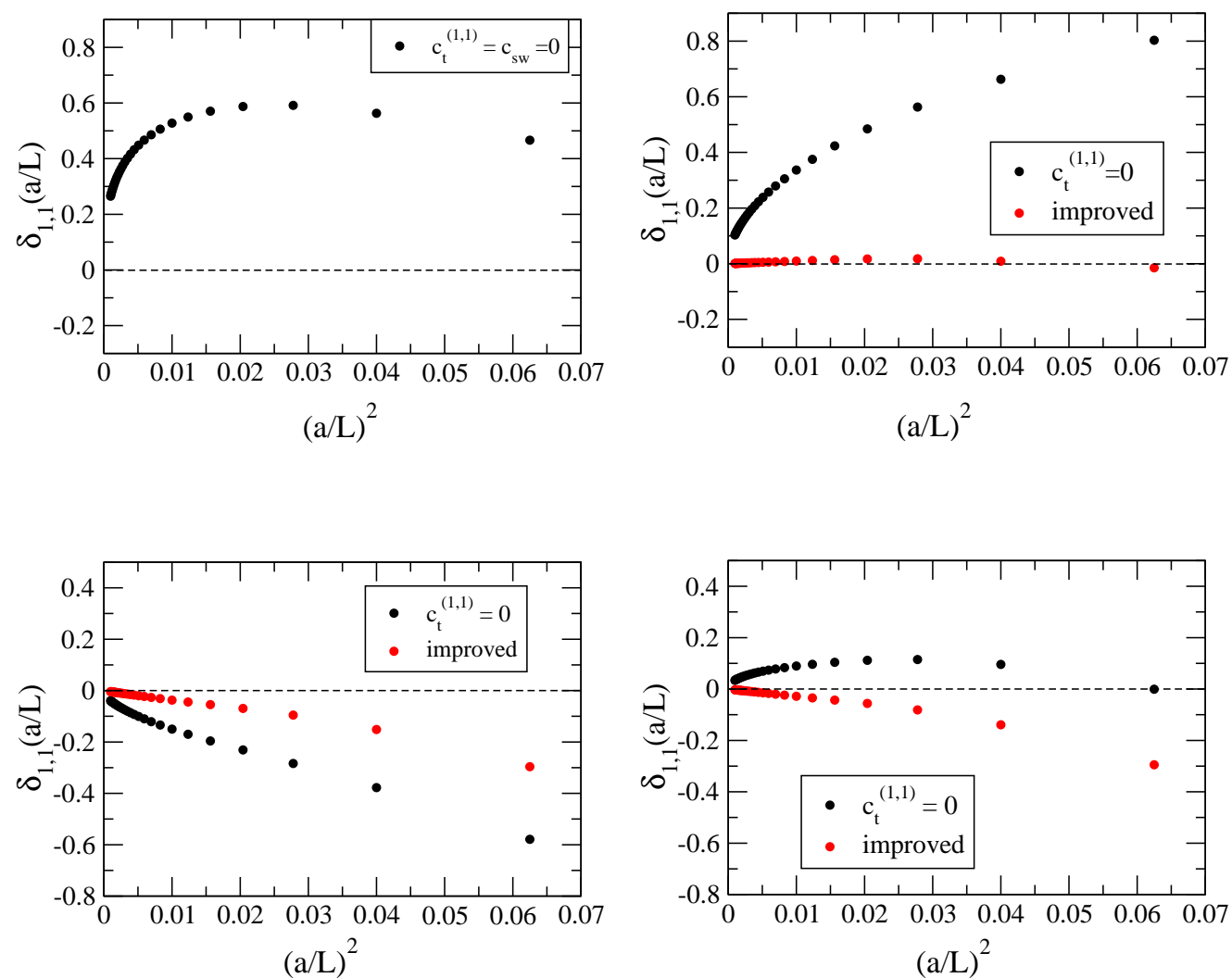

Figure 1: Lattice artefacts $\delta_{1,1}(a / L)$ for fermions in the fundamental representation of SU(3), regularised with the standard SF (upper panels) and the $\chi \mathrm{SF}$ (lower panels), respectively. The 2 panels on the left (right) show data with $c_{\mathrm{sw}}=0\left(c_{\mathrm{sw}}=1\right)$.

of $z_{f}$, a dimension-3 boundary counterterm, in addition to the usual tuning of the bare mass to its critical value $m_{\mathrm{cr}}$. In our perturbative context the critical fermion mass and $z_{f}$ are set to their tree-level values, $m_{\mathrm{cr}}=0$ and $z_{f}=1$. We also assume that tree-level boundary $\mathrm{O}(a)$ improvement is implemented, i.e. we set the tree-level coefficients to $c_{\mathrm{t}}^{(0)}=1, \tilde{c}_{\mathrm{t}}^{(0)}=1, d_{s}^{(0)}=0.5$.

\section{Results}

The asymptotic behaviour of the fermionic one-loop coefficient can be parameterized as follows:

$$
p_{1,1}(L / a) \stackrel{a / L \rightarrow 0}{\sim} r_{0}+s_{0} \ln (L / a)+\frac{a}{L}\left(r_{1}+s_{1} \ln (L / a)\right)+\mathrm{O}\left(a^{2}\right) .
$$

Independently of the regularisation one expects that $s_{0}$ is given by $s_{0}=2 b_{0,1}$. The coefficient $r_{1}$ is cancelled by the correct choice of $c_{\mathrm{t}}^{(1,1)}$ and $s_{1}$ is either proportional to $c_{\mathrm{sw}}-1$ (standard SF) or expected to vanish $(\chi \mathrm{SF})$. We now consider the following cases:

1. standard SF, unimproved $\left(c_{\mathrm{t}}^{(1,1)}=0\right.$ and $\left.c_{\mathrm{sw}}=0\right)$;

2. standard SF, improved in the bulk $\left(c_{\mathrm{sw}}=1\right)$, but not at boundaries $\left(c_{\mathrm{t}}^{(1,1)}=0\right)$; 

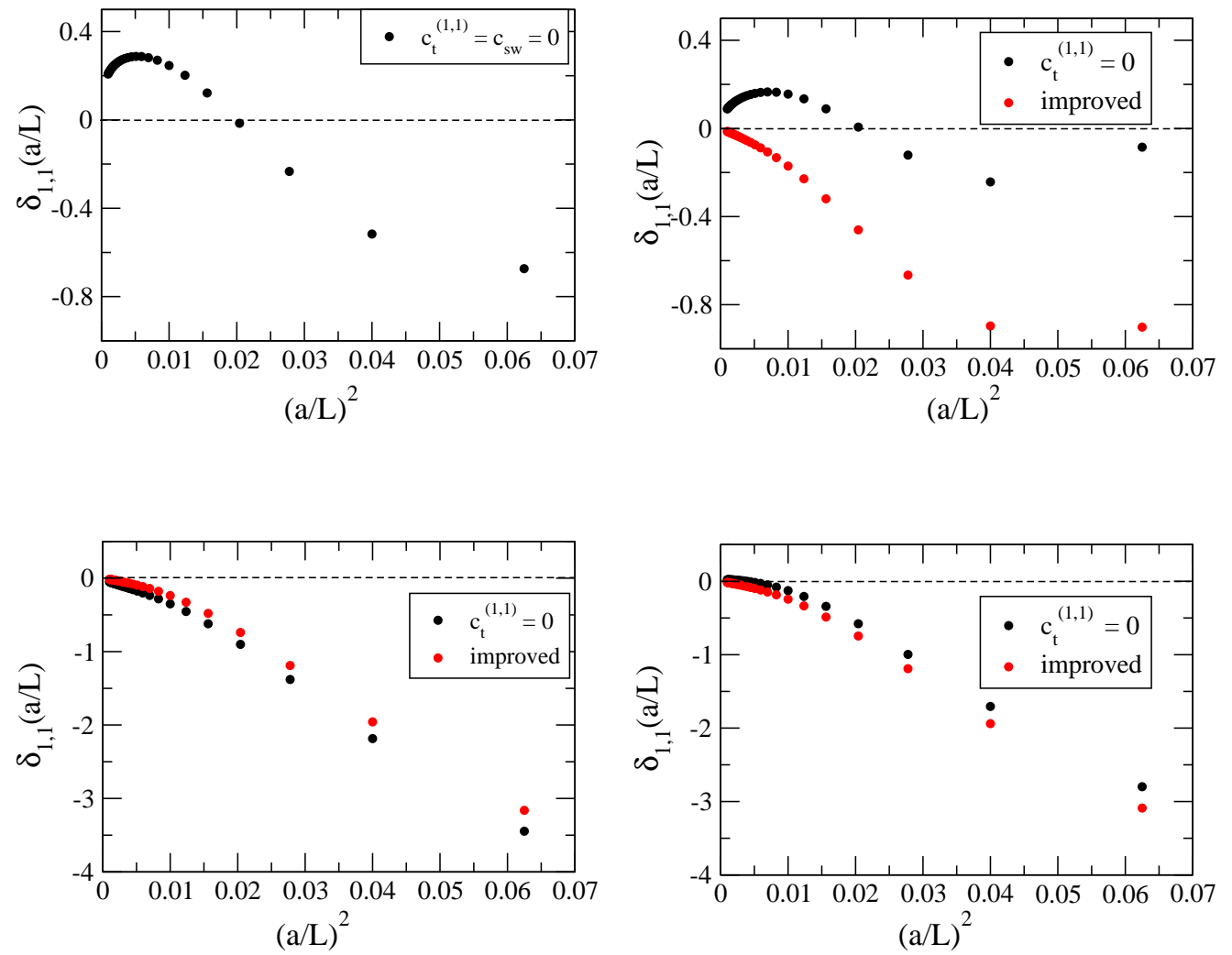

Figure 2: The same as figure 1, for the 2-index symmetric (sextet) representation of SU(3).

3. standard SF, fully improved;

4. $\chi \mathrm{SF}$, unimproved at the boundaries $\left(c_{\mathrm{t}}^{(1,1)}=0\right)$ with either $c_{\mathrm{sw}}=0$ or $c_{\mathrm{sw}}=1$;

5. $\chi \mathrm{SF}$, fully improved, with either $c_{\mathrm{sw}}=0$ or $c_{\mathrm{sw}}=1$.

With fermions in the fundamental representations we see that the cutoff effects are essentially zero in the standard SF, once improvement both in the bulk and at the boundaries is implemented (figure 1). This smallness is likely to be a kinematical accident for this choice of background field and parameters. What is striking, however, is the large size of the effects cancelled by both $c_{\mathrm{sw}}$ and $c_{\mathrm{t}}^{(1,1)}$. In the $\chi \mathrm{SF}$ the situation is as expected: the cutoff effects behave asymptotically as $\mathrm{O}\left(a^{2}\right)$ once the boundary improvement is implemented. We note that the boundary cutoff effect is smaller here and the asymptotic $\mathrm{O}\left(a^{2}\right)$ behaviour is indeed observed (figure 1).

The situation changes drastically if the fundamental representation is replaced by the sextet representation. In this case the cutoff effects are enormous, as illustrated in figure 2 . The situation is bad enough with the standard SF and quite a bit worse with the $\chi \mathrm{SF}$. This is clearly a disaster, and it is obvious that neither framework could be used to extract any sensible continuum results based on data for only a few relatively small lattices.

\subsection{A possible cure: weaken the background field?}

To understand how the problem arises it is useful to recall how the abelian background field 

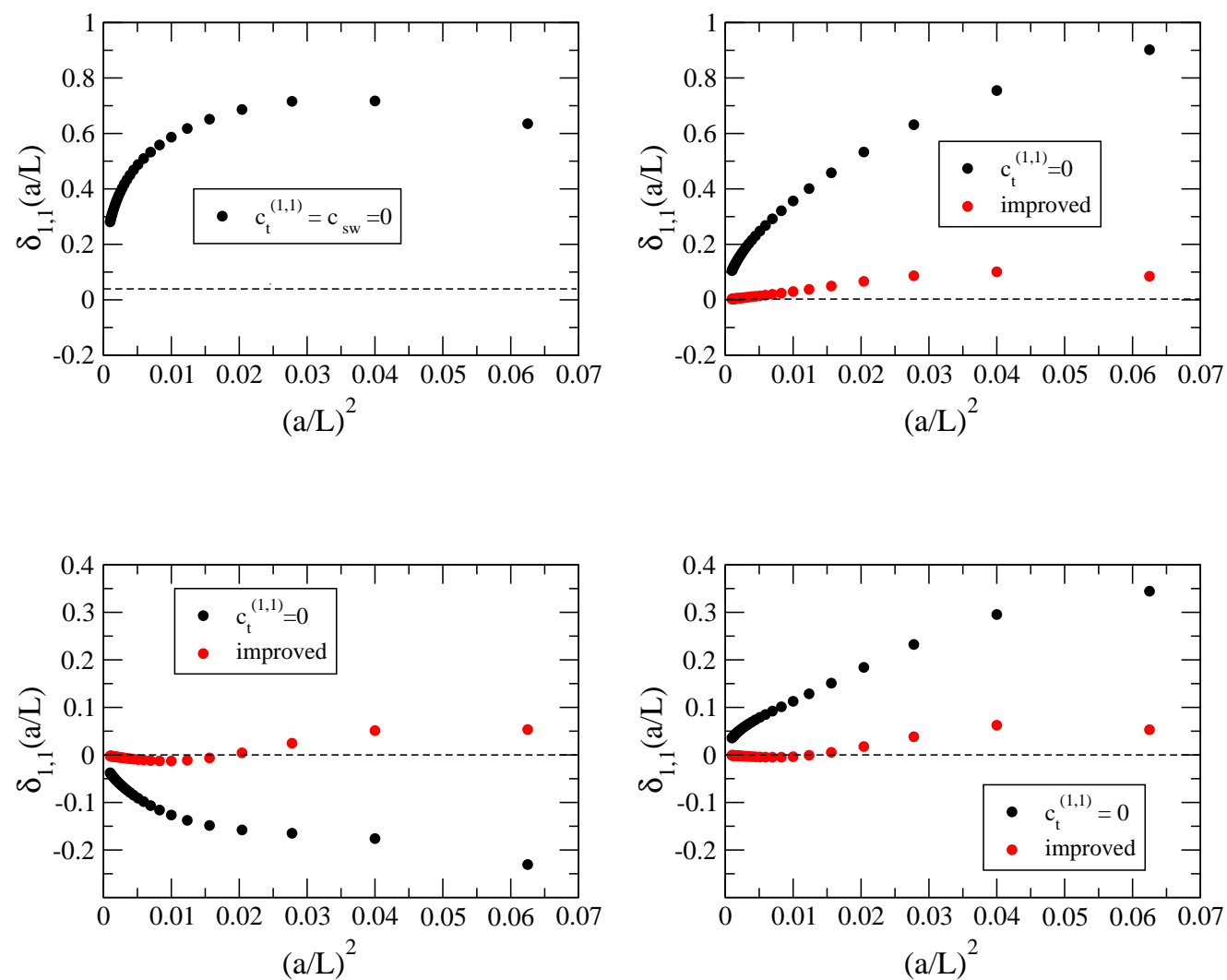

Figure 3: The same as figure 2 with a background field $B$ which is weaker by a factor $1 / 2$.

translates from the fundamental to the symmetric representation. Any given link variable of the SU(3) abelian background field has the form

$$
V^{\mathrm{fun}}(x, \mu)=\exp \left[i \operatorname{diag}\left(\phi_{1}^{f}, \phi_{2}^{f}, \phi_{3}^{f}\right)\right],
$$

and is mapped to a $6 \times 6$ diagonal unitary matrix in the symmetric represenation,

$$
V^{\operatorname{sym}}(x, \mu)=\exp \left[i \operatorname{diag}\left(\phi_{1}^{s}, \phi_{2}^{s}, \phi_{3}^{s}, \phi_{4}^{s}, \phi_{5}^{s}, \phi_{6}^{s}\right)\right],
$$

where the angular variables are related as follows:

$$
\phi_{1}^{s}=2 \phi_{1}^{f}, \quad \phi_{2}^{s}=\phi_{1}^{f}+\phi_{2}^{f}, \quad \phi_{3}^{s}=\phi_{1}^{f}+\phi_{3}^{f}, \quad \phi_{4}^{s}=2 \phi_{2}^{f}, \quad \phi_{5}^{s}=\phi_{2}^{f}+\phi_{3}^{f}, \quad \phi_{6}^{s}=2 \phi_{3}^{f} .
$$

Hence, the fermions in the sextet representation see a background field that is twice as large as for fermions in the fundamental representation. A possible cure could thus be a weaker background field where the boundary gauge fields are halved, so that $B \rightarrow B / 2$. Indeed this reduces the cutoff effects to reasonable levels for the sextet representation, as seen in figure 3 .

\section{Conclusions and future directions}

Our perturbative results are a clear warning for anyone embarking on a non-perturbative study based on the SF coupling. In particular, we find that models with fermions in other than the fundamental representations can suffer from very large cutoff effects even if these are small in QCD 
with otherwise the same parameters ${ }^{1}$. Note that this problem is not cured by a smoothening of the gauge field, as the background field is already smooth and thus would not be changed by a smearing procedure. In particular, our perturbative results are directly relevant for studies such as [15].

It seems that models with fermions in other than the fundamental representations call for a different choice of the background field. Here we have explored a simple minded reduction by a factor $1 / 2$. However, while the cutoff effects in the non-fundamental representations are indeed rendered small, very large effects are now seen with fermions in the fundamental representation (QCD). It is not clear to us whether choices exist with universally small cutoff effects. Note that any modification of the background field requires a re-assessment of the quality of the signal in numerical simulations, as well as a new one-loop calculation for the pure gauge part. A more systematic exploration is currently in progress [11].

Finally, our study establishes that the mechanism of automatic $\mathrm{O}(a)$ improvement works for the SF coupling at one-loop order if regularised with the $\chi \mathrm{SF}$. However, to mimick the situation of a non-perturbative simulation one should determine both $m_{\mathrm{cr}}$ and $z_{f}$ on finite lattices too. This may change the size of residual cutoff effects significantly on the small lattices accessible to numerical simulations [11].

\section{Acknowledgments}

The authors gratefully acknowledge support by SFI under grant 11/RFP/PHY3218 and by the EU unter grant agreement number PITN-GA-2009-238353 (ITN STRONGnet).

\section{References}

[1] J. R. Andersen et al., Eur. Phys. J. Plus 126 (2011) 81

[2] M. Lüscher, R. Narayanan, P. Weisz, U. Wolff, Nucl. Phys. $B 384$ (1992) 168-228.

[3] S. Sint, Nucl. Phys. B421 (1994) 135-158. Nucl. Phys. B451 (1995) 416-444.

[4] L. Del Debbio, PoS LATTICE2010 (2010) 004.

[5] M. Lüscher, S. Sint, R. Sommer, P. Weisz, Nucl. Phys. B478 (1996) 365-400.

[6] M. Lüscher, S. Sint, R. Sommer, P. Weisz, U. Wolff, Nucl. Phys. B491 (1997) 323-343.

[7] T. Karavirta, A. Mykkanen, J. Rantaharju, K. Rummukainen, K. Tuominen, JHEP 1106 (2011) 061.

[8] S. Sint, Nucl. Phys. B847 (2011) 491-531.

[9] S. Sint, B. Leder, PoS LATTICE2010 (2010) 265.

[10] R. Sommer, Nucl. Phys. Proc. Suppl. 60A (1998) 279-294.

[11] P. Vilaseca and S. Sint, in preparation.

[12] M. Lüscher, R. Sommer, P. Weisz, U. Wolff, Nucl. Phys. B413 (1994) 481-502.

[13] S. Sint, R. Sommer, Nucl. Phys. B465 (1996) 71-98.

[14] T. Karavirta, talk at Lattice 2011.

[15] T. DeGrand, Y. Shamir, B. Svetitsky, [arXiv:1110.6845 [hep-lat]].

\footnotetext{
${ }^{1}$ Similar conclusions regarding the standard SF have been reached in [14].
} 